\title{
Research of Image processing in Instant Noodle Packets Auto-Inspection System
}

\author{
Kai Peng ${ }^{12, a}$, Tie-Cheng Wang ${ }^{3, b}$ and Ze-Qing Yang ${ }^{2, c}$ \\ ${ }^{1}$ State key laboratory of precision measuring technology and instruments (Tianjin University), China \\ ${ }^{2}$ School of Mechanical Engineering, Hebei University of technology, China \\ ${ }^{3}$ Engineering Training Center, Hebei University of technology, China. \\ akaip_tju@163.com, bwang.tc@163.com, cyzq82 @163.com
}

Keywords: Image processing; HIS color model; histogram statistics; threshold; recognition

\begin{abstract}
It is good idea that machine vision is introduced to inspect the seasoning packets of instant noodles on-line. The image processing algorithm was a key when the vision system was built up to capture the image of dough with seasoning packets. Image of dough contour was analyzed to find if the dough has a perfect shape. Before pocketing instant noodle, several packets were checked if they lie on the dough by method of histogram statistics of brightness in HIS color model and threshold. Vegetable and oil packets were inspected by the histogram statistics of Hue while the silver salt packet was tested by the histogram statistics of luminance. Testing on actual production line, a system using the image algorithm has the precise identification rate of $100 \%$ and false detection rate of $99 \%$, and the detection speed of better than 20 per minute.
\end{abstract}

\section{Introduction}

Instant noodles represent a fast growing product and have a fast growing market in Asian countries. Since the competition becomes fiercer, instant noodles' profits is sharp decline and instant noodles enterprises confront with more severe challenge [1]. It is more important to improve the quality and varieties of instant noodles for meeting the requirement of different people. In automatic packaging production line, it is not a good way to arrange workers to control the quality of packaging instant noodle. As machine vision is introduced into industry field, the industrial camera is replacing the worker's eyes to observe and measure objects in the scene. Compared with the artificial examination, the machine vision has the merits as the high automaticity, sharp recognition capability, the accuracy measurement and has the widespread application prospect. With the development of machine vision technology and the lower cost of higher capability machine hardware, it is already becoming an important testing role in quality automatic detection about food and the agricultural product. Image processing algorithm is built up in time when images of scene in camera are complex and varied but stable in a special condition [2].

In this paper, a visual inspection system was set up to be placed between the packaging position and packets slide position on the production line which consist of a holder with camera and light source to avoid the impact of existing production lines. The light source was a dome white light which can avoid nearly the noise light outside. The camera was a high speed color camera with $640 \times 480$ resolutions, 60 frames/s and 1394 interface. The system could capture the stable image though the dough moves fast down the camera. The algorithms of image process were tested in the system enough.

\section{Image of dough and packets}

Now the images of instant noodles cake could be captured and saved by the format normally in RGB space when the system was setup with white uniform illumination. A typical image was shown in figure 2.Circular dough lied in the center of image and seasoning packets fell on the dough irregularly. 


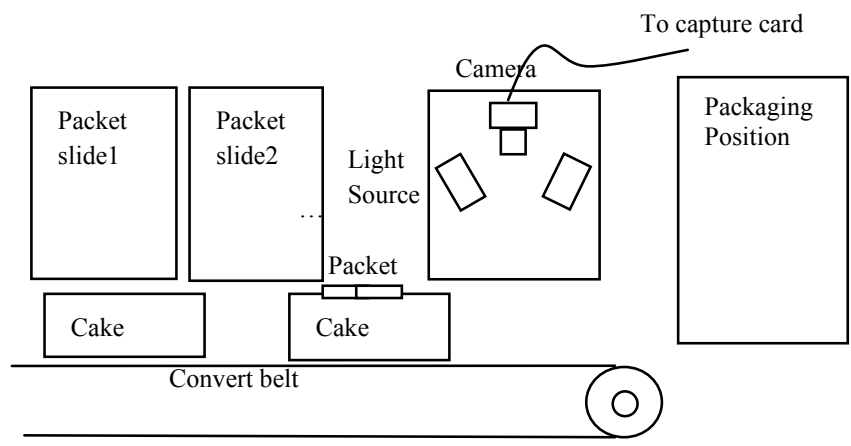

Figure 1. Noodle packet convert system

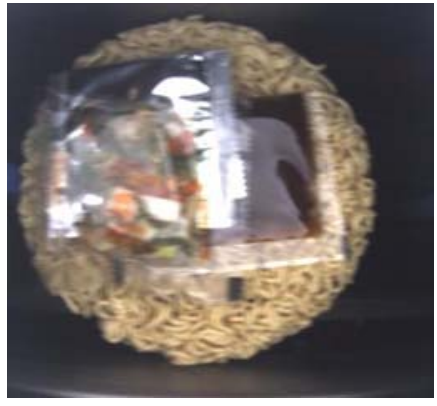

Figure 2. Image of dough and packets

There are generally three types of seasoning packets in the instant noodle, such as salt packet, sauce packet and vegetable packet. Salt packet showed silver color because of the silver packaging paper, sauce packet showed red for the color of sauce and vegetable packet showed green and red because of green dry vegetable and red pepper. The idea of recognizing the packet came from that a worker can make out the different color on eyes.

\section{Preprocessing of Image}

Preprocess is first step in image process that can improve the quality of image and make the object outstanding. First the images need to be change into a gray level one to find the contour of dough easily. As it is shown in figure 3, the pixels with the value of nearly 255 will be thought as the reason of salt packets exit in the gray level image. Of course, the original color image is required to be saved to analysis the component of image color.

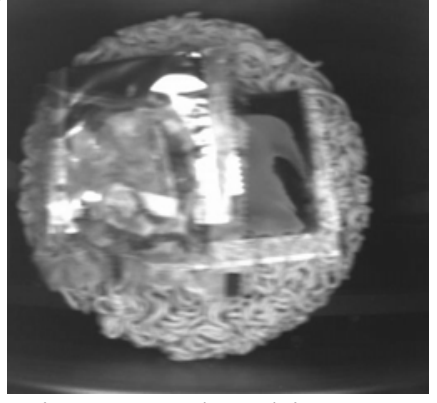

Fig.3 gray level image

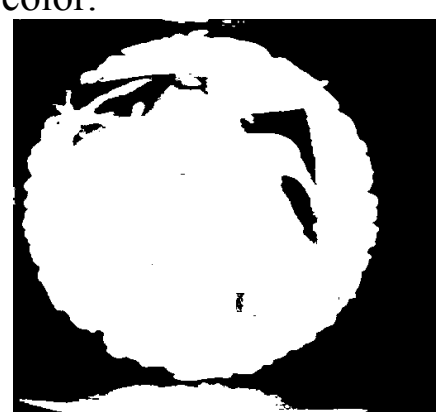

Fig.4 binary image

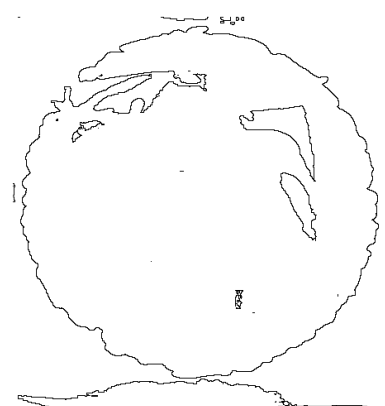

Fig. 5 Extraction of contour

Threshold During the threshold process, individual pixels in an image are marked as object pixels if their value is greater than some threshold value and as background pixels otherwise. Here an object pixel is given a value of " 255 " while a background pixel is given a value of " 0 ." Finally, a binary image shown in figure 4 is created by coloring each pixel white or black, depending on a pixel's labels. The threshold was set on 70 .

\section{Recognition of dough}

In the binary image a large circle pie shown in the centre of image is the dough in eyes. When algorithm is designed to extract the largest contour in the image and to evaluate if the contour is a nearly circle shape. The contour is extracted from the gray level image. The evaluation function of circle shape is shown in eq. 1.

$$
\mathrm{C}=\mathrm{P}^{2} / \mathrm{S}
$$

Shape factor $\mathrm{C}$ is the ratio of the perimeter square $\mathrm{P}^{2}$ and area. The more $\mathrm{C}$ is, the more slender shape is while the minimum of $\mathrm{C}$ is $4 \pi$.

\section{Inspection of packets}

Image processing algorithms is always selected according to the some character of specific target. There is not generally any constant image processing algorithms to deal with a variety of target image. 
In this paper the object was to find if there were the packets on the dough. And the character of seasoning packets was the good basis in pattern recognition in this project.

Color images of instant dough were saved by the format normally in RGB space. An RGB color space is an additive color space which is defined by the three chromaticities of the red, green, and blue additive primaries, and can produce any chromaticity that is the triangle defined by those primary colors. HSI color space is a departure from the human visual system, with the color (Hue), saturation (Saturation or Chroma) and brightness (Intensity or Brightness) to describe the color[3]. Now in the field of image processing and computer vision, there are a number of algorithms easily applied in every component of the HSI color space which can be dealt with separately and are independent of each other. Therefore, the HSI color space can greatly simplify the workload of image analysis and processing [4] [5]

HSI space. HIS is the most common cylindrical-coordinate representations of points in an RGB color model, which rearrange the geometry of RGB in an attempt to be more intuitive and perceptually relevant than the cartesian (cube) representation.

To convert from RGB to HIS

$$
\theta=\cos ^{-1}\left[\frac{(2 * R-G-B) / 2}{\sqrt{(R-G)^{2}+(R-B)(G-B)}}\right], H=\left\{\begin{array}{ll}
\theta & \mathrm{B} \leq \mathrm{G} \\
360-\theta & \mathrm{B}>\mathrm{G}
\end{array}, S=1-\frac{3 \min (R, G, B)}{R+G+B}, I=\frac{R+G+B}{3}\right.
$$

Color Histogram and Packets Recognition. In this scene of camera view, the dough was light yellow, and bright silver was the salt packet, showing a strong reflectivity, a transparent plastic bag with a green leaf and red crushed pepper inside was vegetable packet, and sauce packet was deep red. It was easy to judge by their color if the packets exit.

Histogram describes the number of pixel about the certain property of the image. The horizontal axis is commonly the property level; the vertical axis is the frequency value of the property, which is the number of pixel. In the histogram of intensity (I) coordinate, the histogram was a good double-peak figure and there would be enough pixels above 250 which belonged to silver salt packet. It was shown in Fig.6.

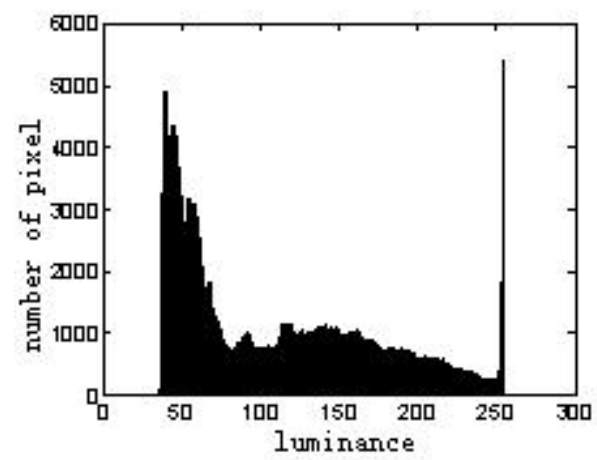

Figure 6. Histogram of luminance

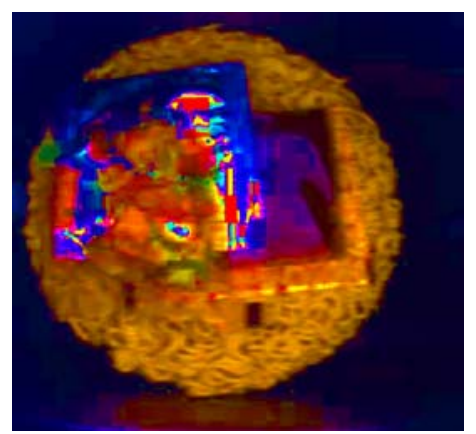

Figure 7. Image of full color

As the color distribution is shown in Fig. 7, the area of the green was little and the area of orange and blue was large. The histogram of $\mathrm{H}$ component o was shown as a typical multi-peak figure in Fig. 8 in which local area enlarged. A large number of pixels were yellow of nearly 60 and belonged to images of dough. The number of green pixels was selected as the basis characteristic color of vegetable packet. And experiment proved that the number of green pixel was a good way to recognize the vegetable packet and the green range is set from 100 to 140. More than 300 green pixels can be determined that there was a vegetable packet exists. The binary image of extracting green pixel was shown in Fig. 10 and black points mark the original green pixel. It was easy to determine whether there was sauce packets exist when the typical red color of sauce packet was large number over 10000 .

\section{Conclusion}


To improve the packets detection on the instant noodle packaging production line, a good inspection system was designed to easily be installed on production lines and easy to promote the use of similar production lines.

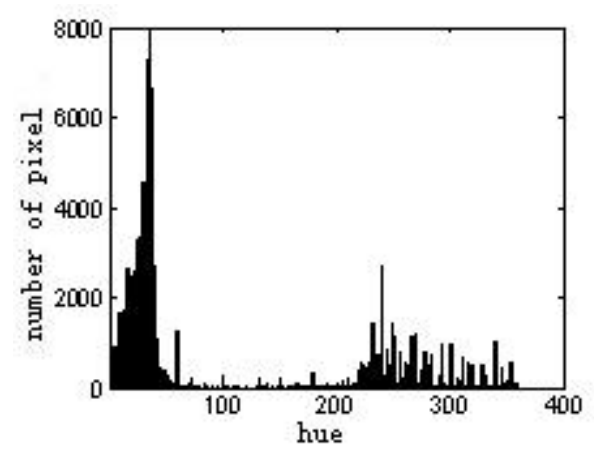

Figure 8. Histogram of hue

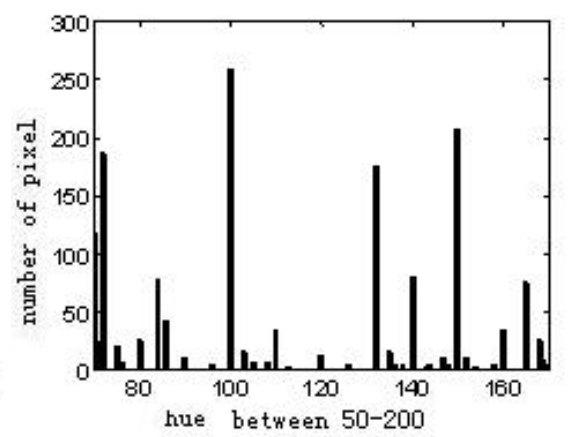

Figure 9. Histogram of local

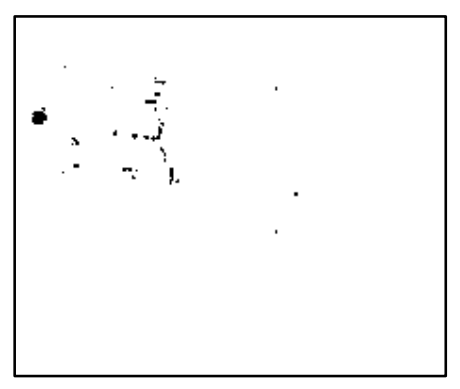
Figure 10. Result of green extraction The HIS space model was applied in the algorithm of the instant image processing and target identification. It overcome the lack of resolution on the RGB color space, improved the system recognition accuracy, and reduced the amount of data image processing to improve the system detection rate.

The inspection system was set up and worked for a long time. The result of long-time work proved that the product quality testing system was high speed, accuracy, and efficiency. The recognition rate of the system was $100 \%$, false detection rate of more than $99 \%$, detection rate of better than 20 packages / min. More advanced are hardware of the visual system, further is the system detection rate.

\section{Acknowledgment}

The authors appreciate the support of State key laboratory of precision measuring technology, instruments and the National Natural Science Foundation of China (Project NO. 50978207), Hebei College Science and Technology Youth Fund NO.2011215

\section{References}

[1] LI Shu-guo, CHEN Hui, LI Xue-mei, REN Yuan-yuan, Current situation,problems and prospect of instant noodle industry in China (Cereals and Oils, 2010)

[2] ZHAO Jie-wen, Chen Zhentao, Zou Xiaobo, machine vision to achieve instant noodles broken line detection( micro-computer information, 2007)

[3] Zhang Quan-hai, Shi Peng-fei, based on the HSV space color image edge detection methods (computer simulation, 2000)

[4] Wang Jian-feng; xiao guoqiang;jiang jian-min, An Image Retrieval Algorithm Based on the HSI Color Space Accumulative Histogram(Computer Engineering and Science,2007)

[5] YIN Jian-jun, WANG Xin-zhong, MAO Han-pin, Contrastive Research on Tomato Image Segmentation Under RGB and HSI Color Space (Journal of Agricultural Mechanization Research, 2006) 